\title{
Modified diffusion-weighted imaging-Alberta Stroke Program Early Computed Tomography Score including deep white matter lesions predicts symptomatic intracerebral hemorrhage following intravenous thrombolysis
}

\author{
Koji Tanaka ${ }^{1}$ (D) . Shoji Matsumoto ${ }^{2,3} \cdot$ Konosuke Furuta $^{2} \cdot$ Takeshi Yamada $^{4} \cdot$ Sukehisa Nagano $^{5} \cdot$ Kei-ichiro Takase $^{6}$. \\ Taketo Hatano ${ }^{7} \cdot$ Ryo Yamasaki ${ }^{1} \cdot$ Jun-ichi Kira ${ }^{1}$
}

Published online: 19 November 2019

(c) The Author(s) 2019

\begin{abstract}
The Alberta Stroke Program Early Computed Tomography Score (ASPECTS) is widely used for the assessment of early ischemic changes (EICs) before thrombolysis. However, for symptomatic intracerebral hemorrhage (sICH) following intravenous recombinant tissue plasminogen activator (rt-PA), the prediction abilities of CT-ASPECTS, diffusion-weighted imaging (DWI)-ASPECTS, and DWI-ASPECTS including EICs in deep white matter (DWI-ASPECTS + W) are unclear. We investigated associations between each score and sICH following intravenous rt-PA. Data from consecutive patients who received intravenous rt-PA for acute ischemic stroke from 2005 to 2015 in four hospitals were retrospectively screened. We included data from patients who had undergone both CT and magnetic resonance imaging before thrombolysis and without evidence of posterior circulation stroke. We analyzed the ability of CT-ASPECTS, DWI-ASPECTS, and DWI-ASPECTS + W to predict sICH, accompanied by an increase in the National Institutes of Health Stroke Scale (NIHSS) score of $\geq 4$ within the initial $36 \mathrm{~h}$. Of 455 patients (273 men, median 75 years old), sICH occurred in 15 patients (3.3\%). Receiver operating characteristics curve analysis showed that the optimal cut-offs of CT-ASPECTS, DWI-ASPECTS, and DWI-ASPECTS + W for predicting sICH were $\leq 9$ (sensitivity $60.0 \%$, specificity $59.8 \%$, c-statistic 0.625 ), $\leq 6$ (sensitivity $53.3 \%$, specificity $80.9 \%$, c-statistic 0.718 ), and $\leq 8$ (sensitivity $86.7 \%$, specificity $55.9 \%$, c-statistic 0.756 ), respectively. A DWI-ASPECTS + W of $\leq 8$ was independently associated with sICH (odds ratio 5.21, 95\% confidence interval 1.30-35.31) after adjustment for pretreatment with antithrombotic agents, pretreatment NIHSS score, and large artery occlusions. DWI-ASPECTS + W predicted sICH in patients with acute anterior circulation stroke receiving intravenous rt-PA.
\end{abstract}

Keywords Symptomatic intracerebral hemorrhage $\cdot$ Thrombolysis · Ischemic stroke · Alberta Stroke Program Early Computed Tomography Score · Diffusion-weighted imaging

Koji Tanaka

tkouji@ neuro.med.kyushu-u.ac.jp

1 Department of Neurology, Neurological Institute, Graduate School of Medical Sciences, Kyushu University, 3-1-1 Maidashi, Higashi-ku, Fukuoka 812-8582, Japan

2 Department of Neurology, Kokura Memorial Hospital, Kitakyushu, Japan

3 Department of Comprehensive Strokology, Fujita Health University School of Medicine, Toyoake, Japan
4 Department of Neurology, Saiseikai Fukuoka General Hospital, Fukuoka, Japan

5 Department of Neurology, Fukuoka City Hospital, Fukuoka, Japan

6 Department of Neurology, Iizuka Hospital, Iizuka, Japan

7 Department of Neurosurgery, Kokura Memorial Hospital, Kitakyushu, Japan 


\section{Highlights}

- The association between Alberta Stroke Program scores (CT-ASPECTS, DWI-ASPECTS, and DWIASPECTS $+\mathrm{W}$ ) and symptomatic intracerebral hemorrhage ( $\mathrm{sICH}$ ) following intravenous recombinant tissue plasminogen activator (rt-PA) has not been investigated.

- DWI-ASPECTS + W predicted sICH more accurately than CT-ASPECTS and DWI-ASPECTS in patients who received intravenous rt-PA for acute ischemic stroke.

- A DWI-ASPECTS + W of $\leq 8$ was independently associated with $\mathrm{sICH}$ after adjustment for pretreatment of antithrombotic agents, severity of stroke symptoms, and large artery occlusions.

- Further research should assess the ability of DWIASPECTS $+\mathrm{W}$ to predict long-term functional outcomes.

\section{Introduction}

Intravenous recombinant tissue plasminogen activator (rtPA) is an established treatment for acute ischemic stroke that reduces mortality and improves long-term functional outcomes. However, intravenous administration of rt-PA increases the risk of sICH [1,2], which leads to high mortality and poor functional outcomes [3]. Early ischemic changes (EICs) on pretreatment computed tomography (CT) or diffusion-weighted imaging (DWI) are predictive for the efficacy of intravenous rt-PA [4-6]. The Alberta Stroke Program Early CT Score (ASPECTS) is a standardized scoring system for the assessment of EICs and is widely used in clinical practice for time-dependent treatment of acute ischemic stroke [7, 8]. The scoring method of ASPECTS has also been applied for the measurement of EICs on DWI with good inter-rater agreement [9-13]. A low DWI-ASPECTS has been identified as predictive for sICH following intravenous rt-PA for acute ischemic stroke $[10,11,14]$.

Recently, Kawano et al. [15] established a modified scoring method, the 11-point DWI-ASPECTS $+\mathrm{W}$, which includes a score of 1 for EICs in deep white matter (WM) in addition to each of the 10 original ASPECTS regions (caudate nucleus, lentiform nucleus, internal capsule, insular ribbon, and M1-6 cortical regions of the middle cerebral artery [MCA]). In that study, both a low DWI-ASPECTS $+\mathrm{W}$ and administration of intravenous rt-PA were predictive for any ICH in patients with acute ischemic stroke, while CT-ASPECTS and DWI-ASPECTS were not. However, in that study, only 36 of 164 patients (22\%) received intravenous rt-PA, and no patients developed sICH following thrombolysis. Therefore, evidence for the association between DWI-ASPECTS $+\mathrm{W}$ and sICH following intravenous rt-PA has been inconclusive. Thus, the purpose of this study was to investigate the ability of CT-ASPECTS, DWI-ASPECTS, and DWI-ASPECTS + W to predict sICH following intravenous rt-PA. We hypothesized that DWI-ASPECTS $+\mathrm{W}$ would more accurately predict sICH in patients receiving intravenous rt-PA than CT-ASPECTS and DWI-ASPECTS.

\section{Methods}

\section{Subjects}

We used data from multicenter retrospective observational study that has been described in detail elsewhere [16]. Briefly, the present study was conducted using data from four urban emergency hospitals with a stroke unit (Saiseikai Fukuoka General Hospital, Fukuoka City Hospital, Iizuka Hospital, and Kokura Memorial Hospital). Subjects in this study comprised consecutive patients who received intravenous rt-PA for acute ischemic stroke between 1 October 2005 and 31 December 2015. All patients received intravenous administration of $0.6 \mathrm{mg} / \mathrm{kg}$ alteplase in accordance with the Japanese guidelines [17]. This study was approved by the Ethics Committees of Kyushu University Hospital (29-111) and each of the facilities.

This study enrolled patients who underwent both CT and magnetic resonance imaging (MRI) including DWI prior to the administration of rt-PA and without evidence of posterior circulation stroke, including EICs in the territory of the vertebrobasilar arteries or posterior cerebral artery and/or occlusion of the basilar artery.

The following clinical information was systematically extracted from medical records: age, sex, vascular risk factors (hypertension, diabetes mellitus, and dyslipidemia), atrial fibrillation, history of stroke, and pretreatment of antithrombotic agents. The pretreatment systolic and diastolic blood pressure, severity of stroke symptoms as assessed by the National Institutes of Health Stroke Scale (NIHSS) score, and onset-to-treatment time were obtained from emergency medical charts. sICH was defined as CT evidence of new parenchymal ICH associated with neurological deterioration that corresponded to an increase of $\geq 4$ points from the baseline NIHSS score within $36 \mathrm{~h}$ after the treatment. 


\section{Assessment of ASPECTS}

Before the intravenous administration of rt-PA, MRI was performed, usually after CT. The time of starting CT and MRI was collected from the electronic time stamp of when the first sequence was acquired. CT scans were performed according to a standard multi-slice CT scan protocol (without contrast enhancement, $120 \mathrm{kV}, 200-270 \mathrm{~mA}, 0.75$ - to 2-s scan time, 5-mm slice thickness, inferior orbitomeatal baseline, and obtained at an appropriate window width and level setting of $\geq 95$ Hounsfield units). MRI scans were performed on a $1.5 \mathrm{~T}$ scanner. MRI protocols were not entirely uniform between hospitals, but all included axial DWI that used single-shot echoplanar imaging (b value, $1000 \mathrm{~s} / \mathrm{mm}^{2}$; slice thickness, 5-7.5 mm; inter-slice gap, 1-1.5 mm).

CT-ASPECTS and DWI-ASPECTS were calculated according to the 10-item ASPECTS lesion method. The 11-point DWI-ASPECTS + W was also calculated by assigning a score of 1 for normal and a score of 0 for EICs in the WM (defined as hyperintense lesions in the corona radiata) in addition to the 10 original ASPECTS regions. The outermost limit of the WM lesion was the subcortex of the M5 region and the innermost limit was the caudate nucleus, which were evaluated at the level $2 \mathrm{~cm}$ superior to the thalamus and striatum, as previously described $[15,18]$. For each score, two experienced physicians ( $\mathrm{K}$ Tanaka and one of the following: SM, TY, SN, or K Takase) independently and retrospectively calculated the CT-ASPECTS, DWI-ASPECTS, and DWI-ASPECTS + W for each hospital. During the imaging interpretations, physicians were blinded to the patients' clinical information and the outcome of the imaging findings. Disagreements were resolved by consensus.

Arterial occlusion sites were assessed using MR angiography, carotid ultrasonography, and/or CT angiography. Large artery occlusions were defined as occlusions of the internal carotid artery or proximal portion of the MCA detected by any modality.

\section{Statistical analysis}

All statistical analyses were performed using JMP statistical software version 9.0 (SAS Institute, Inc., Cary, NC, USA). Data are expressed as the median and interquartile range for continuous variables and the count and percentage for categorical variables. The kappa statistics were used to assess the investigators' agreement about CT-ASPECTS, DWIASPECTS, and WM lesions. Relationships between CTASPECTS, DWI-ASPECTS, and DWI-ASPECTS + W were assessed using a Bland-Altman plot. To obtain the optimal CT-ASPECTS, DWI-ASPECTS, and DWI-ASPECTS + W cut-offs required to predict $\mathrm{SICH}$, receiver operating characteristics curves were constructed. The c-statistics (area under the receiver operating characteristics curve) for the three scores were compared using a nonparametric method [19]. Clinical characteristics between patients with and without sICH were compared using the Chi squared test, Fisher's exact test, or Wilcoxon rank sum test as appropriate. Multivariate logistic regression analysis was performed to assess the independent impact of each cut-off on sICH. Variables with a $p$ value of $<0.05$ in the univariate analysis were included in the multivariate model. A p-value of $<0.05$ was considered statistically significant.

\section{Results}

Overall, 750 consecutive patients who received intravenous rt-PA during the study period were included; of these, 295 were excluded because they had received intravenous rt-PA twice during the study period $(\mathrm{n}=2)$, did not undergo a CT or MRI study ( $n=225)$, had missing data $(n=1)$, or exhibited evidence of posterior circulation stroke $(n=67)$, including EICs in the thalamus $(n=12)$, cerebellum $(n=10)$, brain stem $(n=18)$, posterior cerebral artery territory $(n=8)$, and/ or occlusion of the basilar artery $(n=33)$. Finally, data from 455 patients ( 273 men, median 75 years old) were included in the analysis. The median time from onset of stroke to CT, time delay of MRI after CT, and onset to treatment time was 65 (49-98) min, 23 (14-33) min, and 141 (113-173) min, respectively.

Antithrombotic agents were prescribed to 171 patients (37.6\%) prior to stroke. Antiplatelet therapy (aspirin [n=81], clopidogrel $[\mathrm{n}=12]$, cilostazol $[\mathrm{n}=12]$, and dual antiplatelet therapy $[n=12]$ ) was prescribed to 117 patients. Anticoagulant therapy (warfarin [ $\mathrm{n}=71]$, direct oral anticoagulants $[\mathrm{n}=3]$, and unfractionated heparin $[\mathrm{n}=3]$ ) was prescribed to 77 patients. Twenty-three patients received a combination of antiplatelet and anticoagulant therapy. The median CT-ASPECTS, DWI-ASPECTS, and DWI-ASPECTS + W scores were 10 (9-10), 8 (7-9), and 9 (8-10), respectively. WM lesions were seen in 255 patients $(56.0 \%)$, and the CT-ASPECTS and DWI-ASPECTS scores were lower in patients with WM lesions ( 10 [8-10] vs. 10 [9-10], $\mathrm{p}=0.003$ and 8 [7-10] vs. 9 [8-10], p $<0.001$, respectively). Large artery occlusions were seen in 227 (51.0\%) of 445 patients who were evaluated for arterial occlusion sites before thrombolysis, including the internal carotid artery $(\mathrm{n}=86)$ and the proximal portion of the MCA $(n=141)$. Sixty-three patients (13.8\%) underwent endovascular therapy alongside thrombolysis.

The weighted kappa statistics of agreement between the physicians for CT-ASPECTS and DWI-ASPECTS were 0.482 and 0.696 , respectively. The kappa statistic for the presence of WM lesions was 0.618. Figure 1 shows the Bland-Altman plots of differences between CT-ASPECTS vs. DWI-ASPECTS and CT-ASPECTS vs. 


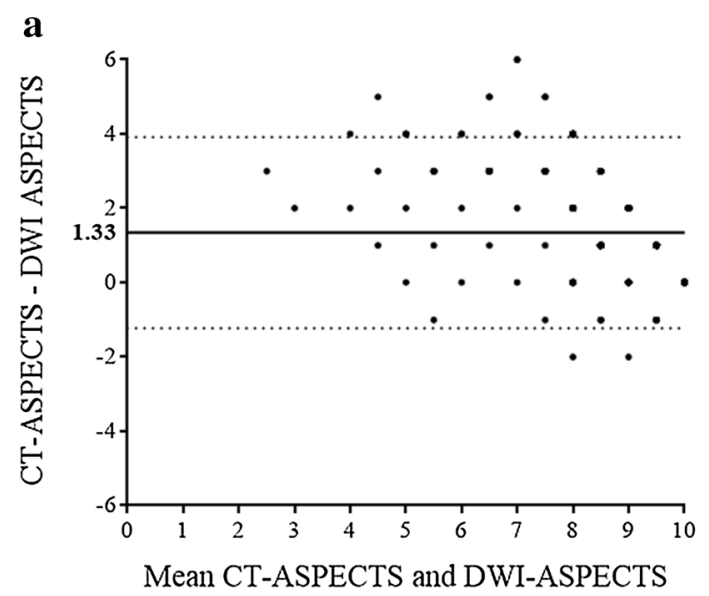

Fig. 1 Bland-Altman plots of Alberta Stroke Program scores. a The mean difference between CT-ASPECTS and DWI-ASPECTS was 1.33 . b The mean difference between CT-ASPECTS and DWIASPECTS $+\mathrm{W}$ was 0.77 . The horizontal line shows the mean dif-

DWI-ASPECTS $+\mathrm{W}$. The mean difference between CTASPECTS and DWI-ASPECTS was 1.33 , and the mean difference between CT-ASPECTS and DWI-ASPECTS + W was 0.77 .

Of the 455 patients, 15 (3.3\%) had sICH. Compared with patients without $\mathrm{SICH}$, patients with sICH more frequently received pretreatment with antithrombotic agents $(66.7 \%$ vs. $36.6 \%, p=0.027)$, had a higher pretreatment NIHSS score (median 21 vs. $15, \mathrm{p}=0.003$ ), and more frequently

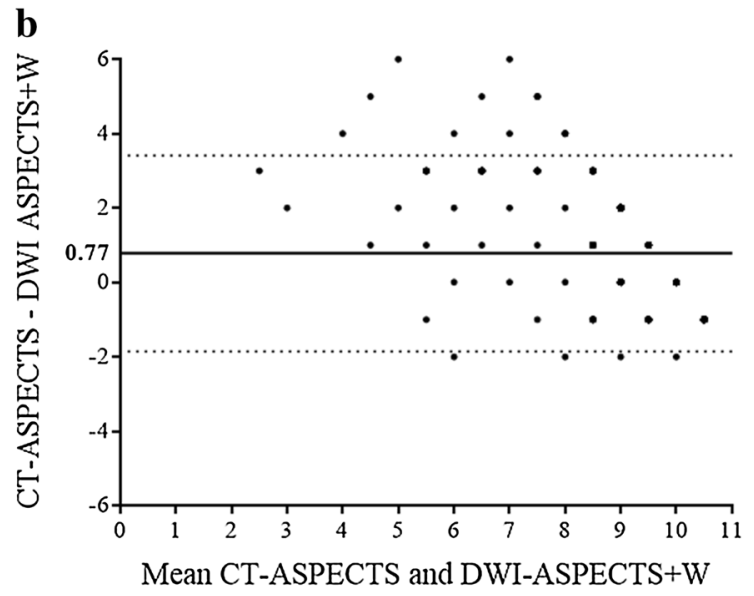

ference in scores, and the dotted lines show the standard deviations. ASPECTS Alberta Stroke Program Early Computed Tomography Score, DWI diffusion-weighted imaging

had large artery occlusion ( $80.0 \%$ vs. $50.0 \%, \mathrm{p}=0.033)$. The DWI-ASPECTS (median 6 vs. $8, \mathrm{p}=0.004$ ) and DWIASPECTS $+\mathrm{W}$ (median 7 vs. $9, \mathrm{p}<0.001$ ) scores were lower in patients with sICH than those without (Table 1). For predicting sICH, the optimal cut-offs of CT-ASPECTS, DWI-ASPECTS, and DWI-ASPECTS $+\mathrm{W}$ were $\leq 9$ (sensitivity $60.0 \%$, specificity $59.8 \%$, c-statistic 0.625 ), $\leq 6$ (sensitivity $53.3 \%$, specificity $80.9 \%$, c-statistic 0.718 ), and $\leq 8$ (sensitivity $86.7 \%$, specificity $55.9 \%$, c-statistic 0.756 ),

Table 1 Univariate analysis of clinical characteristics of patients with and without symptomatic intracerebral hemorrhage

\begin{tabular}{|c|c|c|c|c|}
\hline Variable & Total $\mathrm{N}=455$ & With sICH N $=15$ & Without $\mathrm{sICH} N=440$ & p-value \\
\hline Sex, male* & $273(60.0)$ & $9(60.0)$ & $264(60.0)$ & 1.000 \\
\hline Age (years) $\dagger$ & $75(65-82)$ & $76(67-87)$ & $75(65-82)$ & 0.481 \\
\hline Hypertension* & $308(67.7)$ & $10(66.7)$ & $298(67.7)$ & 1.000 \\
\hline Dyslipidemia* & $121(26.6)$ & $4(26.7)$ & $117(26.6)$ & 1.000 \\
\hline Diabetes mellitus* & $98(21.5)$ & $6(40.0)$ & $92(20.9)$ & 0.104 \\
\hline Atrial fibrillation* & $243(53.4)$ & $10(66.7)$ & $233(53.0)$ & 0.431 \\
\hline Pretreatment of antithrombotic agents* & $171(37.6)$ & $10(66.7)$ & $161(36.6)$ & 0.027 \\
\hline Systolic blood pressure $(\mathrm{mmHg}) \dagger$ & $159(141-180)$ & $158(150-171)$ & $159(140-180)$ & 0.738 \\
\hline Diastolic blood pressure $(\mathrm{mmHg}) \dagger$ & $86(74-100)$ & $84(76-94)$ & $86(74-100)$ & 0.854 \\
\hline Pretreatment NIHSS score $\dagger$ & $16(10-21)$ & $21(17-26)$ & $15(10-20.75)$ & 0.003 \\
\hline CT-ASPECTS $\dagger$ & $10(9-10)$ & $10(8-10)$ & $10(9-10)$ & 0.064 \\
\hline DWI-ASPECTS $\dagger$ & $8(7-9)$ & $6(4-8)$ & $8(7-9)$ & 0.004 \\
\hline DWI-ASPECTS + W† & $9(8-10)$ & $7(5-8)$ & $9(8-10)$ & $<0.001$ \\
\hline Large artery occlusions $(\mathrm{N}=445)^{*}$ & $227(51.0)$ & $12(80.0)$ & $215(50.0)$ & 0.033 \\
\hline Onset to treatment time $(\min ) \dagger$ & $141(113-173)$ & $130(100-164)$ & $141(113-174)$ & 0.507 \\
\hline Endovascular therapy* & $63(13.8)$ & $0(0)$ & $63(14.3)$ & 0.243 \\
\hline
\end{tabular}

Data are presented as $\mathrm{N}(\%)$ or median (interquartile range)

NIHSS National Institutes of Health Stroke Scale, ASPECTS Alberta Stroke Program Early Computed Tomography Score, DWI diffusion weighted imaging

*Fisher's exact test, ${ }^{\dagger}$ Wilcoxon rank sum test 
Table 2 Receiver-operating-characteristic curve analysis of Alberta Stroke Program scores for predicting symptomatic intracerebral hemorrhage

\begin{tabular}{|c|c|c|c|c|c|c|}
\hline Variable & c-statistic $(95 \% \mathrm{CI})$ & Optimal cutoff & Sensitivity (\%) & Specificity (\%) & PPV (\%) & NPV $(\%)$ \\
\hline CT-ASPECTS & $0.625(0.468-0.759)$ & $\leq 9$ & 60.0 & 59.8 & 3.6 & 97.8 \\
\hline DWI-ASPECTS & $0.718(0.565-0.833)$ & $\leq 6$ & 53.3 & 80.9 & 8.7 & 98.1 \\
\hline DWI-ASPECTS + W & $0.756(0.629-0.850)$ & $\leq 8$ & 86.7 & 55.9 & 6.3 & 99.2 \\
\hline
\end{tabular}

ASPECTS Alberta Stroke Program Early Computed Tomography Score, $C I$ confidence interval, $P P V$ positive predictive value, $N P V$ negative predictive value, $D W I$ diffusion weighted imaging

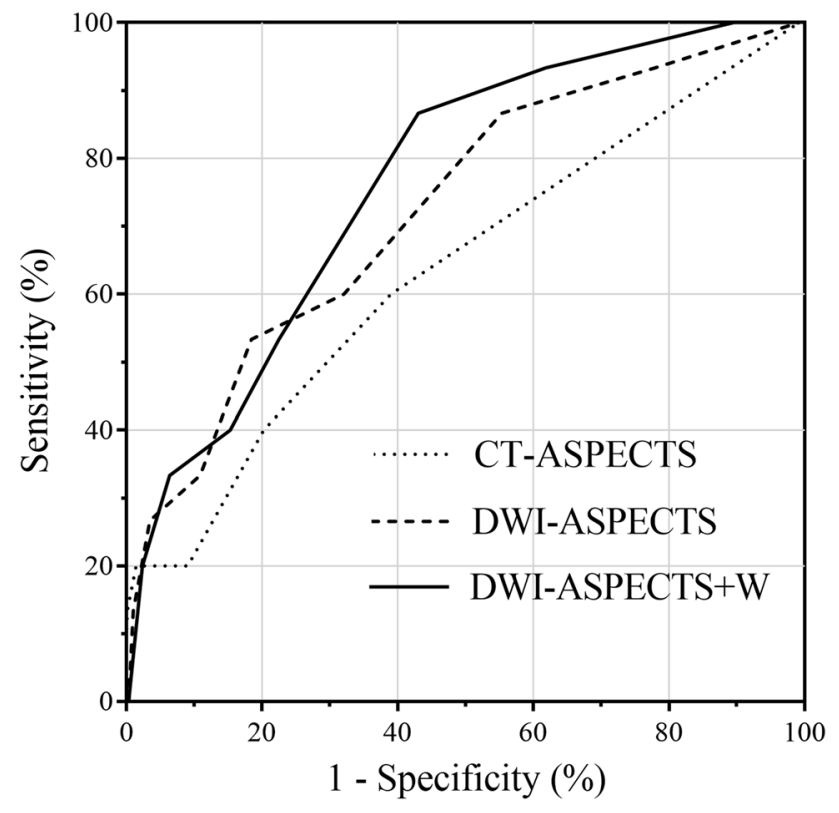

Fig. 2 Receiver operating characteristics curves of Alberta Stroke Program scores for predicting symptomatic intracerebral hemorrhage. There was a significant difference in the c-statistics among the three scores $(p=0.039)$, and DWI-ASPECTS $+\mathrm{W}$ was superior to CT-ASPECTS $(\mathrm{p}=0.032)$ and DWI-ASPECTS $(\mathrm{p}=0.040)$ in predicting symptomatic intracerebral hemorrhage. ASPECTS Alberta Stroke Program Early Computed Tomography Score, DWI diffusionweighted imaging

Table 3 Multivariate logistic regression analysis of cut-offs of Alberta Stroke Program scores for predicting symptomatic intracerebral hemorrhage

\begin{tabular}{lll}
\hline Variable & Odds ratio $(95 \% \mathrm{CI})$ & p-value \\
\hline CT-ASPECTS $\leq 9$ & $1.25(0.39-3.81)$ & 0.694 \\
DWI-ASPECTS $\leq 6$ & $2.72(0.89-8.56)$ & 0.079 \\
DWI-ASPECTS $+\mathrm{W} \leq 8$ & $5.21(1.30-35.31)$ & 0.018 \\
\hline
\end{tabular}

ASPECTS Alberta Stroke Program Early Computed Tomography Score, $C I$ confidence interval, $D W I$ diffusion weighted imaging

The models were adjusted for pretreatment of antithrombotic agents, pretreatment National Institutes of Health Stroke Scale score, and large artery occlusions respectively (Table 2). There was a significant difference in the $\mathrm{c}$-statistic among the three scores $(\mathrm{p}=0.039)$, and DWIASPECTS $+\mathrm{W}$ was superior to CT-ASPECTS $(\mathrm{p}=0.032)$ and DWI-ASPECTS $(\mathrm{p}=0.040)$ for predicting sICH (Fig. 2).

The multivariate logistic regression analysis revealed that a DWI-ASPECTS $+\mathrm{W}$ of $\leq 8$ was significantly associated with sICH (odds ratio 5.21, 95\% confidence interval 1.30-35.31) after adjustment for pretreatment of antithrombotic agents, pretreatment NIHSS score, and large artery occlusions (Table 3 ).

\section{Discussion}

This study investigated the association between the extent of EIC as measured by three scores and sICH following intravenous rt-PA. Among the three scores, DWI-ASPECTS + W was superior to both CT-ASPECTS and DWI-ASPECTS in predicting sICH. This study showed that the inter-rater agreement was good for DWI-ASPECTS and moderate for CT-ASPECTS. This better inter-rater agreement on DWI than CT is consistent with that in previous studies $[9,11,12$, 13]. The mean DWI-ASPECTS was 1.33 points lower than the mean CT-ASPECTS. This discrepancy between DWIASPECTS and CT-ASPECTS scores was larger than in previous studies, which reported a mean difference of 0.43 [20] in ischemic stroke patients within $7 \mathrm{~h}$ of onset, a median difference of 1 point [21] in stroke patients within $24 \mathrm{~h}$ of onset, and a mean difference of 0.92 in stroke patients within $3 \mathrm{~h}$ of onset [11]. There may be two possible reasons for this finding. First, the median time from stroke onset to CT in this study was $65 \mathrm{~min}$, which was relatively shorter than that in the previous studies; the mean and median times from symptom onset to CT were $117 \mathrm{~min}$ and $2.7 \mathrm{~h}[20,21]$. Therefore, some EICs might have been overlooked before becoming apparent on CT. Second, the median time delay of DWI after CT was $23 \mathrm{~min}$, which was slightly longer than that of 19 min previously reported in a study with patients who had received intravenous rt-PA [11]. Alongside the higher sensitivity of DWI than CT for detecting EICs, some EICs became apparent after $\mathrm{CT}$. 
This study showed that the c-statistics for DWIASPECTS $+\mathrm{W}$ are higher than for CT-ASPECTS and DWIASPECTS for predicting sICH following intravenous rt-PA. This indicates that WM lesions, which are often difficult to detect on CT, can be a precursor to the development of sICH. The corona radiata consists of various WM tracts and is mainly fed by the deep lenticulostriate branches of the MCA [22]. Subcortical WM has higher thresholds for infarctions than does gray matter [23-25]. Therefore, WM lesions indicate more severe ischemia in the MCA territory. This is also supported by our finding that patients with WM lesions had lower CT-ASPECTS and DWI-ASPECTS than those without WM lesions. Kawano et al. [18] reported that the presence of WM lesions was predictive for the absence of early dramatic improvement after intravenous rt-PA. An increased risk of sICH in patients with WM lesions would partially explain the negative correlation between WM lesions and early dramatic improvement.

The cut-off value of DWI-ASPECTS $+\mathrm{W}$ was independently associated with sICH following intravenous rt-PA. The cut-off value, a DWI-ASPECTS $+\mathrm{W}$ of $\leq 8$, was 1 point higher than that reported in a previous study [15], in which the DWI-ASPECTS $+\mathrm{W}$ cut-off value for predicting any ICH was $\leq 7$. This might be because all participants in this study received intravenous rt-PA, and the elevated risk of bleeding lowered the threshold of sICH. Our results indicate that patients with a DWI-ASPECTS $+\mathrm{W}$ of $\geq 9$ can be administered rt-PA safely. Although recent American Heart Association guidelines recommend non-contrast $\mathrm{CT}$ as an imaging modality prior to intravenous rt-PA [26], and the utility of CT cannot be denied, our results indicate that DWI is more useful for predicting sICH following intravenous rt-PA. Moreover, the 11-point DWI-ASPECTS + W, which simply adds information on WM lesions to the 10 ASPECTS regions, was able to more accurately predict which patients were prone to $\mathrm{sICH}$ in the acute phase than the original ASPECTS based on both CT and DWI.

This study has several limitations. First, this was a retrospective study with a limited number of patients, which might have led to statistical errors. Second, the imaging modality before thrombolysis was decided by an independent attending physician. This might contribute to selection bias in patients who underwent both CT and DWI. Moreover, performing both CT and DWI before thrombolysis might lead to some delay in treatment and affect the outcome. Third, post-treatment parameters that could have affected the incidence of sICH (e.g. blood pressure, recanalization of the occluded artery, and antithrombotic agents used after the initial $24 \mathrm{~h}$ ) were not included. Further research including these factors will inform the development of preventative measures for sICH following intravenous rt-PA in patients with low ASPECTS. Finally, associations between each ASPECTS and long-term functional outcomes were unclear because we did not use the 3-month modified Rankin scale in the present study.

In conclusion, DWI-ASPECTS + W was able to predict sICH following intravenous rt-PA more accurately than CTASPECTS and DWI-ASPECTS. DWI-ASPECTS $+\mathrm{W}$ is a promising method for predicting sICH following intravenous rt-PA in patients with anterior circulation stroke.

Acknowledgements We would like to thank the Center for Clinical and Translational Research, Kyushu University, for the maintenance and management of the Research Electronic Data Capture database. We thank Dr. Chie Kikutake, Medical Institutes of Bioregulation, Kyushu University, for advice on statistical analyses. We thank Nia Cason, $\mathrm{PhD}$, from Edanz Group (www.edanzediting.com/ac) for editing a draft of this manuscript.

Funding This study was supported by the Japan Society for the Promotion of Science Grant-in-Aid for Scientific Research, Grant Number 16K10727 and Grant-in-Aid for Research Activity Start-up, Grant Number 19K21303.

\section{Compliance with ethical standards}

Conflict of interest The authors declare that they have no conflicts of interest.

Open Access This article is distributed under the terms of the Creative Commons Attribution 4.0 International License (http://creativeco mmons.org/licenses/by/4.0/), which permits unrestricted use, distribution, and reproduction in any medium, provided you give appropriate credit to the original author(s) and the source, provide a link to the Creative Commons license, and indicate if changes were made.

\section{References}

1. National Institute of Neurological Disorders and Stroke rt-PA Stroke Study Group (1995) Tissue plasminogen activator for acute ischemic stroke. N Engl J Med 333:1581-1587

2. Wahlgren N, Ahmed N, Dávalos A et al (2007) Thrombolysis with alteplase for acute ischaemic stroke in the Safe Implementation of Thrombolysis in Stroke-Monitoring Study (SITS-MOST): an observational study. Lancet 369:275-282

3. Strbian D, Sairanen T, Meretoja A et al (2011) Patient outcomes from symptomatic intracerebral hemorrhage after stroke thrombolysis. Neurology 77:341-348

4. Hacke W, Kaste M, Fieschi C et al (1995) Intravenous thrombolysis with recombinant tissue plasminogen activator for acute hemispheric stroke. The European Cooperative Acute Stroke Study (ECASS). JAMA 274:1017-1025

5. Moulin T, Cattin F, Crépin-Leblond T et al (1996) Early CT signs in acute middle cerebral artery infarction: predictive value for subsequent infarct locations and outcome. Neurology 47:366-375

6. Nighoghossian N, Hermier M, Adeleine P et al (2003) Baseline magnetic resonance imaging parameters and stroke outcome in patients treated by intravenous tissue plasminogen activator. Stroke 34:458-463

7. Barber PA, Demchuk AM, Zhang J et al (2000) Validity and reliability of a quantitative computed tomography score in predicting outcome of hyperacute stroke before thrombolytic therapy: 
ASPECTS Study Group Alberta Stroke Programme Early CT Score. Lancet 355:1670-1674

8. Schröder J, Thomalla G (2017) A critical review of Alberta Stroke Program Early CT Score for evaluation of acute stroke imaging. Front Neurol 7:245

9. Fiebach JB, Schellinger PD, Jansen O et al (2002) CT and diffusion-weighted MR imaging in randomized order: diffusionweighted imaging results in higher accuracy and lower interrater variability in the diagnosis of hyperacute ischemic stroke. Stroke 33:2206-2210

10. Singer OC, Kurre W, Humpich MC et al (2009) Risk assessment of symptomatic intracerebral hemorrhage after thrombolysis using DWI-ASPECTS. Stroke 40:2743-2748

11. Nezu T, Koga M, Nakagawara J et al (2011) Early ischemic change on CT versus diffusion-weighted imaging for patients with stroke receiving intravenous recombinant tissue-type plasminogen activator therapy: Stroke Acute Management with Urgent Risk-Factor Assessment and Improvement (SAMURAI) rt-PA Registry. Stroke 42:2196-2200

12. Mitomi M, Kimura K, Aoki J et al (2014) Comparison of CT and DWI findings in ischemic stroke patients within 3 hours of onset. J Stroke Cerebrovasc Dis 23:37-42

13. McTaggart RA, Jovin TG, Lansberg MG et al (2015) Alberta Stroke Program early computed tomographic scoring performance in a series of patients undergoing computed tomography and MRI: reader agreement, modality agreement, and outcome prediction. Stroke 46:407-412

14. Nezu T, Koga M, Kimura K et al (2010) Pretreatment ASPECTS on DWI predicts 3-month outcome following rt-PA: SAMURAI rt-PA Registry. Neurology 75:555-561

15. Kawano H, Hirano T, Nakajima M et al (2012) Modified ASPECTS for DWI including deep white matter lesions predicts subsequent intracranial hemorrhage. J Neurol 259:2045-2052

16. Tanaka K, Matsumoto S, Yamada S et al (2019) Temporal trends in clinical characteristics and door-to-needle time in patients receiving intravenous tissue plasminogen activator: a retrospective study of four hospitals in Japan. J Stroke Cerebrovasc Dis 28:104305

17. Minematsu K, Toyoda K, Hirano T et al (2013) Guidelines for the intravenous application of recombinant tissue-type plasminogen activator (alteplase), the second edition, October 2012: a guideline from the Japan Stroke Society. J Stroke Cerebrovasc Dis 22:571-600
18. Kawano H, Hirano T, Inatomi $Y$ et al (2010) Presence of deep white matter lesions on diffusion-weighted imaging is a negative predictor of early dramatic improvement after intravenous tissue plasminogen activator thrombolysis. Cerebrovasc Dis 30:230-236

19. DeLong ER, DeLong DM, Clarke-Pearson DL (1988) Comparing the areas under two or more correlated receiver operating characteristic curves: a nonparametric approach. Biometrics 44:837-845

20. Barber PA, Hill MD, Eliasziw M et al (2005) Imaging of the brain in acute ischaemic stroke: comparison of computed tomography and magnetic resonance diffusion-weighted imaging. J Neurol Neurosurg Psychiatry 76:1528-1533

21. Kosior RK, Lauzon ML, Steffenhagen N et al (2010) Atlas-based topographical scoring for magnetic resonance imaging of acute stroke. Stroke 41:455-460

22. Sharma VK, Wong LK (2016) Middle cerebral artery disease. In: Grotta JC, Albers GW, Broderick JP et al (eds) Stroke: pathophysiology, diagnosis, and management, 6th edn. Elsevier, Philadelphia, pp 362-392

23. Bristow MS, Simon JE, Brown RA et al (2005) MR perfusion and diffusion in acute ischemic stroke: human gray and white matter have different thresholds for infarction. J Cereb Blood Flow Metab 25:1280-1287

24. Arakawa S, Wright PM, Koga M et al (2006) Ischemic thresholds for gray and white matter: a diffusion and perfusion magnetic resonance study. Stroke 37:1211-1216

25. Chen C, Bivard A, Lin L et al (2019) Thresholds for infarction vary between gray matter and white matter in acute ischemic stroke: a CT perfusion study. J Cereb Blood Flow Metab 39:536-546

26. Powers WJ, Rabinstein AA, Ackerson T et al (2018) 2018 Guidelines for the early management of patients with acute ischemic stroke: a guideline for healthcare professionals from the American Heart Association/American Stroke Association. Stroke 49:e46-e110

Publisher's Note Springer Nature remains neutral with regard to jurisdictional claims in published maps and institutional affiliations. 